\title{
An Improved Evaluation System of Physical Education Balanced Development based on Distributed SVM Data Mining Algorithm
}

\author{
Benyu $\mathrm{Xi}^{1}$ and Lina Gong ${ }^{2}$ \\ ${ }^{1}$ City College, Xi'an Jiaotong University, Xi'an 710049, Shaanxi, China \\ ${ }^{2} X i$ 'an Physical Education University, Xi'an 710068, Shaanxi, China \\ wdyx727@163.com
}

\begin{abstract}
Support vector machine (SVM) is an important algorithm in data mining; it can transform the nonlinear classification problem into a linear classification problem by increasing the dimension of the data. The author points out the shortcomings of the traditional data analysis methods, and puts forward the method of complex simulation data analysis based on distributed SVM data mining algorithm. In the empirical part, through construct the evaluation index system of the school sports balanced development mode, the results show that the primary indicators of the sports balanced development are resource allocation(0.3774), school physical education process(0.2781), school physical education results(0.2450), and school sports social environment(0.1000).Overall, the balanced development of school physical education is a long and gradual process, sports evaluation index system also needs to be constantly updated and revised.
\end{abstract}

Keywords: SVM algorithm, data mining, physical education, index system

\section{Introduction}

With the development of network technology, the network has entered into the field of physical education, which has opened up a new way for the reform of physical education. Under the guidance of the new technology, the auxiliary teaching of the sports network course is also carried out in full swing [1]. In the new technology environment, how to sports talents culture, how to overcome the problems faced by the sports network curriculum development, how to construct a universal sports network teaching platform model, the course as long as according to the program design requirements and add the teaching content, you can generate a door can be automatically updated to the sports network curriculum, these are placed in a new issue in the field of sports scientific research, pending further research and practice [2-3]. Network education resources are generally the increase of traditional education resources, upgrading, and regeneration and become, the construction of network resources is the process of resource regeneration. Educational resources include educational theory, educational management and educational resources in general. Such as electronic lesson plans, electronic courseware and electronic library. In the aspect of college physical education, it is to be widely used in sports education network information technology, to promote the development of college sports network education [4]. However, compared with the requirements of modern network information technology development, there is still a certain gap in the development of sports network education resources in China. Therefore, strengthening the analysis of China's college sports network education resources construction situation, correctly guide the construction and development of network education resources have become an important task of current our country college sports network education information.

Educational equity is the foundation of social equity, and the balanced development of compulsory education is a necessary means to promote educational equity. School 
physical education is an important part of education, so the balanced development of education also includes the balanced development of school physical education [5]. Education fairness and education balanced development theory is the theoretical basis of the balanced development of school physical education, balanced development of school physical education theory connotation and balanced education is come down in one continuous line, the balanced development of school sports include different levels, the intercollegiate equilibrium is the foundation of regional, urban and rural balanced, so the school level is the basic level of balanced development of school physical education evaluation [6]. Promoting social fairness and justice and build socialism harmonious society's essential mission and core values, social justice contains fair content of different areas, such as fair income, health equity, education fair, and so on, the educational justice is the foundation of social justice system, if the education fairness cannot be achieved, social equity is lost premise and guarantee [7-8]. Therefore, under the historical background of building a socialist harmonious society in our country, only to constantly promote the balanced development of education in order to gradually realize the education fair, in order to provide a realistic basis for the realization of social fairness. The balanced development of compulsory education in recent years, the research focus in the educational circles, this paper on the analysis and deduction of education fairness and education balanced development theory, expounds the connotation of the theory of balanced development of school physical education, this paper puts forward and balanced development of school physical education evaluation index system is constructed based on the concept of inter school level of the balanced development of school physical education and the empirical research, this paper to enrich the school sports basic theory, promote balanced development of school physical education has a certain theoretical and practical value.

\section{Data Mining and Support Vector Machine (SVM)}

\subsection{Structural Risk Minimization}

Data mining is one of the steps in data knowledge discovery (Databases KnowledgeDiscovery, KDD); it generally refers to the process of searching for information hiding in the data from a large number of data.

\section{Data Mining: A Knowledge Discovery (KDD) Process}

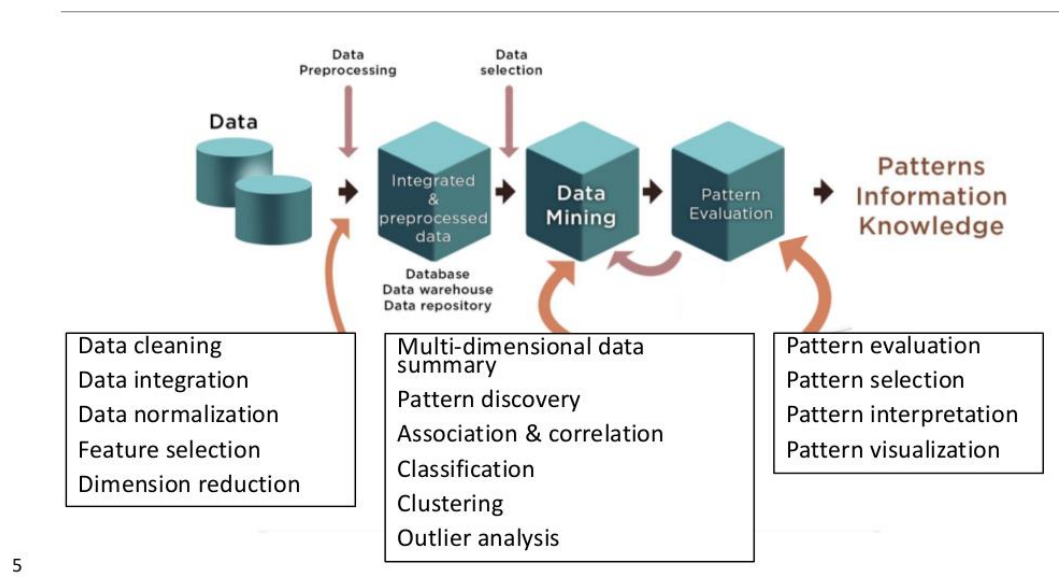

Figure 1. The Knowledge Discovery Process 
Data mining is not an alternative to traditional statistical analysis techniques. Instead, he is an extension and extension of statistical analysis methodology. Most of the statistical analysis techniques are based on the perfect mathematical theory and superb skills, the accuracy of prediction is satisfactory, but the user's requirements are very high. With the increasing of computing power, it is possible for us to use the computer's powerful computing power to accomplish the same function only through the relatively simple and fixed methods. Some of the emerging technologies also in knowledge discovery field has achieved very good results, such as neural network and decision tree, in plenty of data and computation ability, they almost do not have to care of automatically completed many valuable functions. Data mining is the use of the application of statistical and artificial intelligence techniques, he put these profound complex technology package, so that people do not own master these techniques can complete the same function, and focus more on their own to solve the problem.

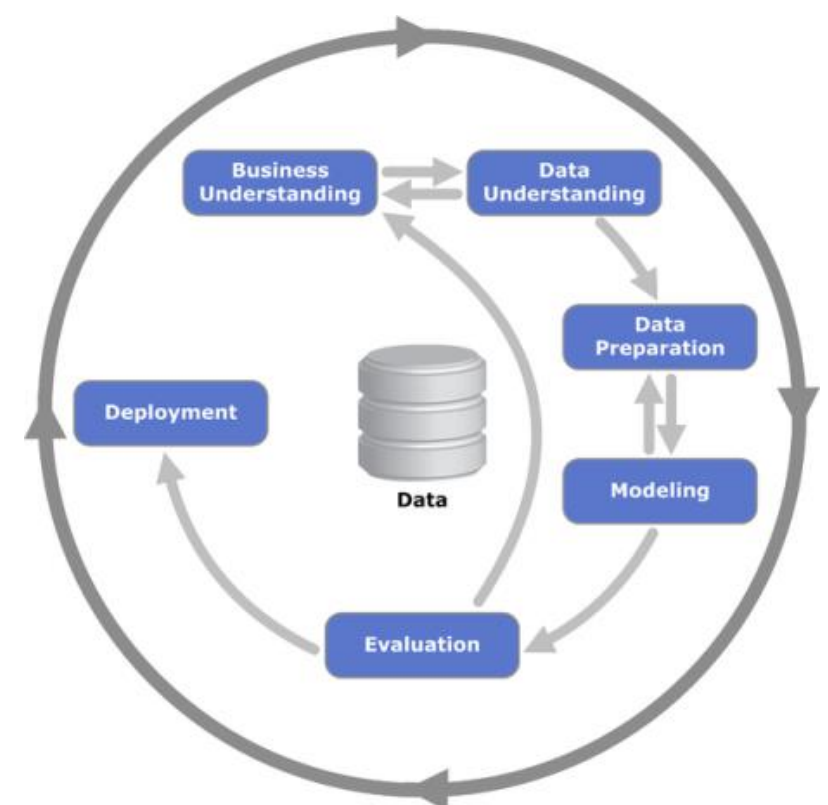

Figure 2. The Data Mining Process

\subsection{Structural Risk Minimization}

Support vector machine is a new machine learning method based on statistical learning theory, and it is a statistical machine learning theory developed in the case of small samples. SVM learning problem can be expressed as a convex optimization problem, so we can find the global minimum value of the objective function by the known effective algorithm. The generalization ability of SVM is very strong, and the noise in training data has little influence on the training of the classifier. The disadvantage of SVM is that the selection of kernel function and parameters is difficult, and there is no clear selection rule. 


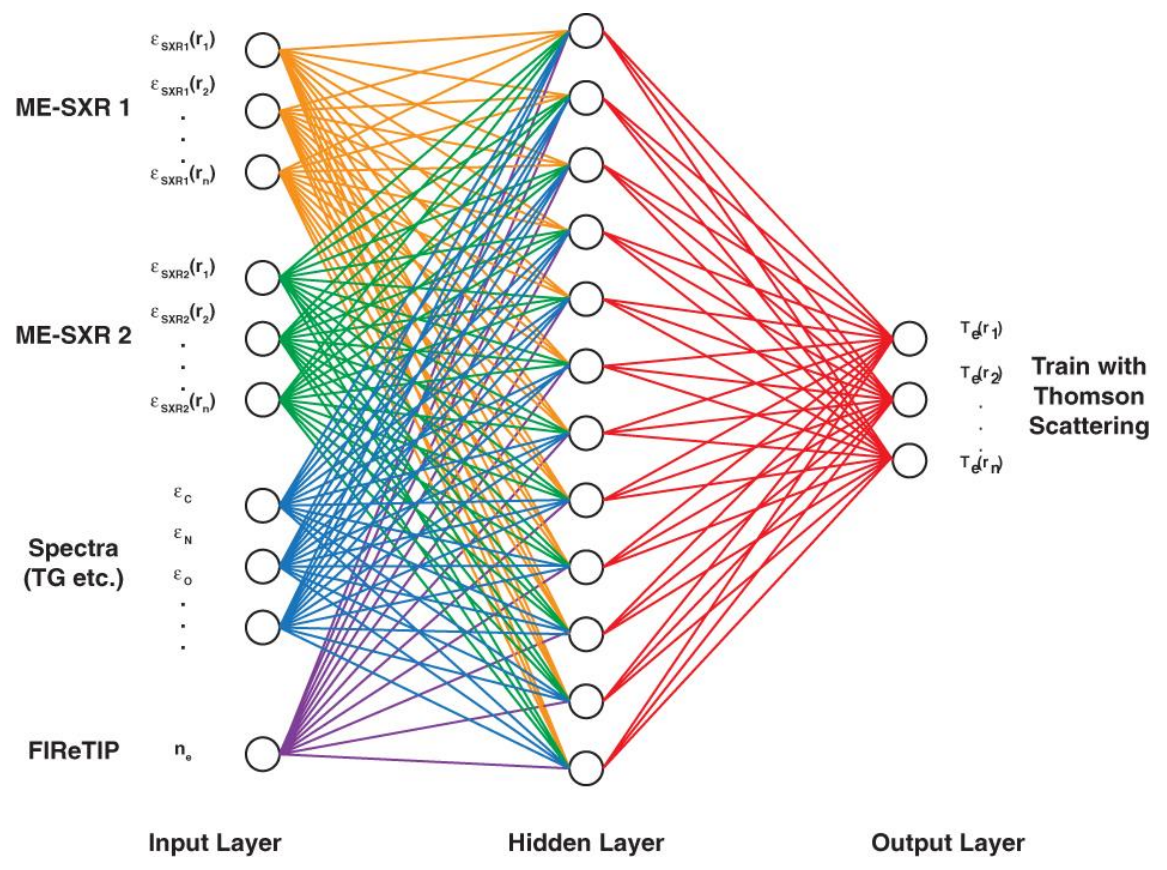

Figure 3. Neural Network Model

Based on the sample machine learning, in fact, it is a link between the input and output through the training samples. In order to find an optimal function $f\left(\mathrm{x}, \pi_{0}\right)$ to approximate the links between the input $\mathrm{x}$ and the output $\mathrm{y}$ and theory requirements follows the minimization of the expected risk:

$$
R(\pi)=\int L(y, f(x, \pi)) d F(x, y)
$$

In formula (1), the probability density function is unknown, so the above expectation risk cannot be directly calculated. Therefore, the expected risk of $\mathrm{R}(\pi)$ to replace the experience of risk:

$$
R_{\text {emp }}(\pi)=\frac{1}{l} \sum_{i=1}^{l} L\left(y_{i}, f\left(x_{i}, \pi\right)\right)
$$

If the expectations of the risk $\mathrm{R}(\pi)$ and empirical risk $\mathrm{R}_{\text {emp }}(\pi)$ according to the convergence of probability $p$ to the same limit inf $R(\pi)$ is called empirical risk minimization principle is consistent.

Empirical risk minimization does not necessarily converge to the minimum expected risk, that is, the empirical risk minimization principle is not always established. To this end, Vapnik after in-depth study put forward the principle of structural risk minimization (SRM), that is:

$$
R(\pi) \leq R_{\text {emp }}(\pi)+\sqrt{\frac{h \ln \left(\frac{2 l}{h}\right)-\ln \left(\frac{\eta}{4}\right)}{l}}
$$

Among them, 1 is the number of sample points, $\eta$ parameters $(0 \leq \eta \leq 1), h$ is a function of the dimension of $\mathrm{f}$, referred to as $\mathrm{VC}$ dimension. 


\subsection{Support Vector Machine Model}

If all the vectors in the training set can be correctly classified by a certain hyper plane, and the nearest vector distance between the planes is the largest, that is, the edge is maximized. In the linear separable model, classification of SVM in a $\mathrm{H}$ inner space structure for super plane:

$$
\langle w, x\rangle+b=0, \quad w \in H, b \in R
$$

Here $\mathrm{w}$ is an adjustable weight vector, and is perpendicular to the hyper plane, $\mathrm{b}$ is the partial value. The formula corresponds to the decision function.

$$
f(x)=\operatorname{sgn}\langle\langle w, x\rangle+b\rangle
$$

For linearly separable sets of samples, the total can be adjusted by $w$ and $b$ :

$$
\begin{aligned}
& \langle w, x\rangle+b \geq 1, y=1 \\
& \langle w, x\rangle+b \leq-1, y=-1
\end{aligned}
$$

Obviously, this adjustment does not change the discriminant function (5). Define two standard canonical hyper planes as:

$$
\langle w, x\rangle+b=1 \quad \text { and } \quad\langle w, x\rangle+b=-1
$$

The sample points on the two standard hyper planes are called support vectors, and the name of the support vector machine is obtained. Define the classification interval (margin) as:

$$
\rho=2 r=\frac{2}{\|w\|}
$$

As a result, the optimization problem for linearly separable SVM is:

$$
\min J(w, b)=\frac{1}{2}\|w\|^{2}
$$

The above problem is the main problem of SVM, which is a convex two optimization problem. The problem can be solved by constructing the Lagrange multiplier method to solve the problem.

$$
\begin{gathered}
\max W(\alpha)=\sum_{j=1}^{l} \alpha_{j}-\frac{1}{2} \sum_{i . j=1}^{l} \alpha_{i} \alpha_{j} y_{i} y_{j} x_{i} x_{j} \\
\sum_{j=1}^{l} \alpha_{i} y_{j}=0
\end{gathered}
$$




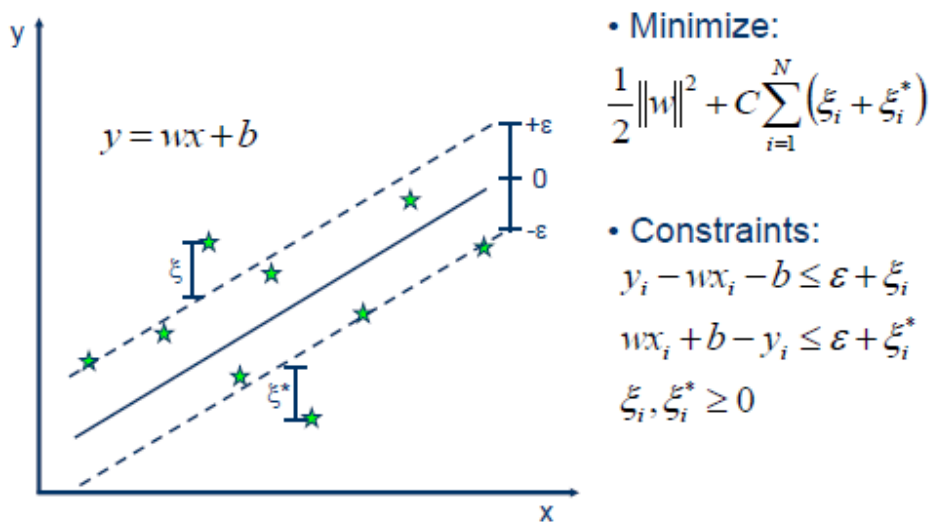

Figure 4. SVM Classification

\section{Sports Balanced Development Evaluation Index}

\subsection{Index System}

Construction of evaluation index system is the theoretical premise of evaluation activities. In addition, the balanced development of school physical education in the implementation of the evaluation of the basic object is to investigate a school. Therefore, it is necessary to establish a school of the evaluation objects, in order to be able to reflect the school physical education balanced development status of evaluation index system of value content. Before the establishment of evaluation index system, the first step is to construct the index system of the theoretical model: model theory is the basis and foundation for construction of evaluation index system of, it reflects the index system on the content, structure and level of scientific and reasonable, is to construct evaluation index system of the early necessary theoretical results. School physical education is an important part of education, and according to the world economic cooperation organization (OECD) practice, the education system model is in accordance with the education background, input, process, results. The four part is divided into two parts. In addition, the principle of educational equity is the theoretical basis of the balanced development of education.

After establishing the theoretical model of the evaluation index system, the next step of theoretical preparation is to further the content of the evaluation of the specific, hierarchical: that is, the establishment of evaluation index system. The establishment of the index system can be divided into two steps: the initial establishment of the evaluation index system and the improvement of the evaluation index system. Evaluation index system of the preliminary establishment needs some preparations in the theoretical premise down, when the researchers to evaluate the field with the breadth and depth of theoretical understanding, according to the purpose of evaluation, the index system was established, the main task of this stage is through the system analysis method to construct the framework of evaluation index system. The main methods used to build the evaluation index system are comprehensive method, analysis method, cross method and index attribute grouping method. Initially established the evaluation index system is not perfect, because of which may contain some unnecessary indexes, and in the structure may also exist at the same level index logical relationship between chaos, not independent of each other; also, it is possible to because of the theoretical preparation or the cause enough experience of lack of some sensitive indicators need to be incorporated into the. Therefore, we must continue to deal with the initial establishment of the index system, so that it is further improved. 


\subsection{Construction Principle}

Evaluation index system is the key part of the evaluation activities, it is the job evaluation, the actual operation of the ruler, so whether to conduct a reasonable and effective evaluation so as to realize the target evaluation depends first and foremost on the evaluation index system is scientific and reasonable, only use the relative scientific reasonable evaluation index system to make the evaluation results more accurate and effective. Therefore, in order to establish a more scientific and systematic index system, to evaluate the current situation of the balanced development of school sports, give full play to the evaluation index system description, monitoring, comparison and evaluation of the function.

1) Scientific principle: scientific principle is any academic research activities must follow the fundamental principles, science is evaluation index system based on the theory of fully, according to reasonable. Only in this way can accurately reflect the connotation of the balanced development of school sports. Implement the scientific principle first requirements to ensure that the theoretical basis of the evaluation of the correctness and rationality, the theoretical basis of the study of the evaluation index system is education fairness and education system model, model of educational fairness and education system is the evaluation index system construction basis and content sources.

2) Overall principle: the important content of school physical education is the education, and is a part of sports, so the balanced development of school physical education evaluation is a comprehensive evaluation, which includes not only the content of educational evaluation, from the subject characteristics, it also belongs to sports field evaluation. In the evaluation of the balanced development of school sports, should try to achieve both the two evaluation perspective, at the same time to highlight the theoretical connotation of educational equity, multi-level, comprehensive reflection of the current situation and level of school sports balanced development.

3) Key principles: the target system in the selection process, to choose a representative core and key indexes, our specific requirements should be focused in the design index, rather than to all relevant factors are listed, all the factors are not on the index set to pile gun together theory the characteristics of the index system designed to highlight the evaluation index system, to clear the status quo is to measure the balanced development of school physical education, instead of the conventional evaluation of school physical education to carry out, so the conventional indicators of some school physical education evaluation, in sensitivity under the guidance of the principle to the theory with the characteristics of equilibrium the development of evaluation for the standard to choose the index of hoof,

4) Operating principle: the principle of operation, first of all in the process in the early period of the construction of evaluation index system shall fully consider each of the indicators of testability, to observable indicator of quantitative or qualitative measurement can be mutual comparison, has a practical significance for evaluation; in addition, evaluation index is not the more the better, too complex and cumbersome index system not only difficult to highlight the evaluation of the main content and key, in actual operation may also evaluation because of the difficulty of data acquisition and cannot be implemented. Therefore, considering the scientific, comprehensive and sensitive indicators, we must also do everything from the reality

5) Principle of combining quantitative and qualitative: evaluation index can be divided into qualitative index and quantitative quality two categories according to their properties: characteristics of qualitative indicators is usually it can accurately reflect the essence of the evaluation, evaluation of the evaluation object can help 
the value level, quality, condition and other aspects of the analysis and judgment of nature the evaluation involves people's feelings, attitude and status on the aspects of qualitative indicators can give full play to people's initiative, get more real information, but the disadvantage is that easy to be subjective, objectivity weaker; quantitative index is the index of digital and quantitative, more objective and accurate, but also conducive to the evaluation of the calculation and grading work.

\section{Empirical Analysis}

\subsection{Index Construction}

On analysis of balanced education theory and education equality principle, through the preliminary interviews with experts on how to in a balanced education within the theoretical framework established to reflect the evaluation index system of balanced development of school physical education status quo won the important information, collect information, under the guidance of the principles of a balanced development of school physical education evaluation index system, the initial establishment of the evaluation index system of balanced development of school physical education. The initial establishment of school physical education balanced development evaluation index system, level indicators total five, respectively is: school sports resource allocation index, school sports management indicators, school sports process indicators, school sports results index and the school sports community environmental indicators.

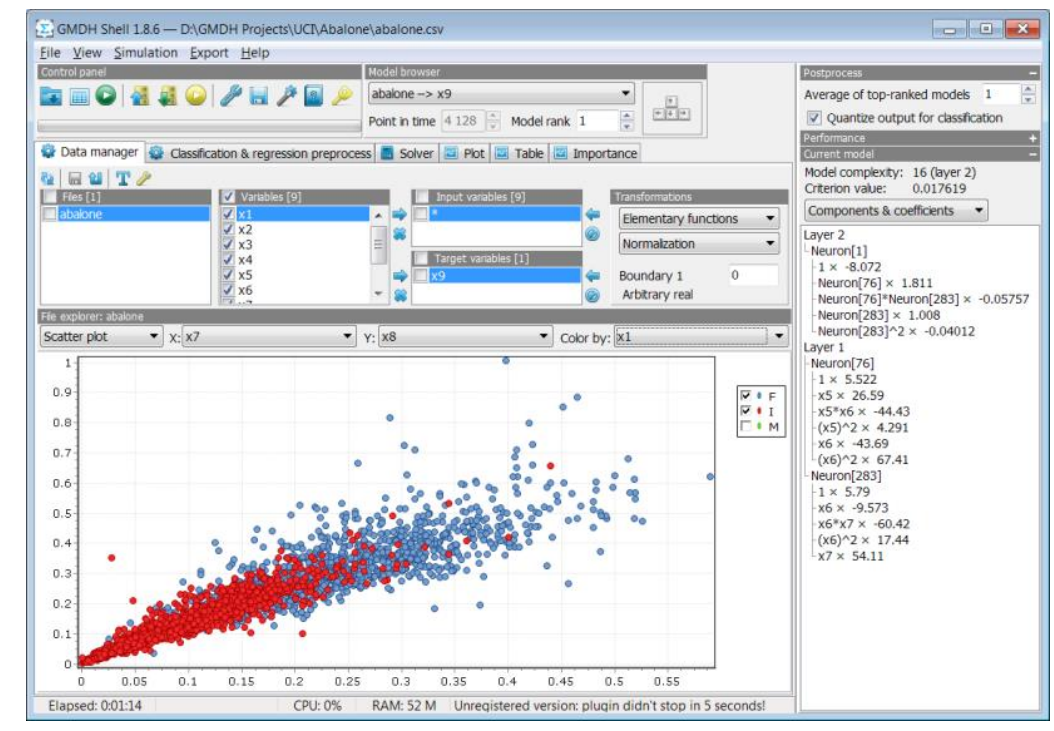

Figure 5. Data Mining Software Analysis

Education resource is refers to the investment sum in the education process of human, material and financial resources, including funding for education, teaching staff and administrative staff, educational facilities, equipment and other series and education related, tangible or intangible resources. Physical education is a part of school education, so the concept of the deduction, the school sports resources defined as investment in school sports in the work of the human, financial and material resources of the total. Then according to the three items of human, financial and material, the school sports resources are further divided into three parts, the school sports funds, the school sports facilities and physical education teachers. 
Table 1. Primary Indicators of Sports Balanced Development

\begin{tabular}{|c|c|c|}
\hline serial & Index name & Pass rate \\
\hline 1 & Resource allocation & $100 \%$ \\
\hline 2 & School Physical Education Management & $95 \%$ \\
\hline 3 & School physical education process & $100 \%$ \\
\hline 4 & School physical education results & $100 \%$ \\
\hline 5 & School sports social environment & $95 \%$ \\
\hline
\end{tabular}

Table 2. Secondary Indicators of Sports Balanced Development

\begin{tabular}{|c|c|c|c|}
\hline serial & primary indicators & secondary indicators & Pass rate \\
\hline \multirow{4}{*}{1} & \multirow{4}{*}{ Resource allocation } & funds & $100 \%$ \\
\hline & & field & $100 \%$ \\
\hline & & equipment & $100 \%$ \\
\hline & & information resources & $85 \%$ \\
\hline \multirow{4}{*}{2} & \multirow{4}{*}{$\begin{array}{c}\text { School Physical Education } \\
\text { Management }\end{array}$} & Our Faculty & $100 \%$ \\
\hline & & Organizational institution & $100 \%$ \\
\hline & & Working mechanism & $100 \%$ \\
\hline & & Sports policy & $100 \%$ \\
\hline \multirow{3}{*}{3} & \multirow{3}{*}{$\begin{array}{c}\text { School physical education } \\
\text { process }\end{array}$} & $\begin{array}{c}\text { Curriculum } \\
\text { implementation }\end{array}$ & $90 \%$ \\
\hline & & physical education & $90 \%$ \\
\hline & & Spare time sports & $100 \%$ \\
\hline \multirow{3}{*}{4} & \multirow{3}{*}{$\begin{array}{l}\text { School physical education } \\
\text { results }\end{array}$} & Work effect & $95 \%$ \\
\hline & & Cultivation of talents & $75 \%$ \\
\hline & & Sports accomplishment & $95 \%$ \\
\hline \multirow{3}{*}{5} & \multirow{3}{*}{$\begin{array}{l}\text { School sports social } \\
\text { environment }\end{array}$} & sports culture & $95 \%$ \\
\hline & & Family influence & $100 \%$ \\
\hline & & Gymnasium & $95 \%$ \\
\hline
\end{tabular}

\subsection{Parameter Test}

As mentioned above, this study through the first round of expert investigation on the index system to adjust, delete and add the work, and now on the basis of the second round of the expert questionnaire. And on the second round of expert investigation results are mathematical statistics, quantitative analysis methods to determine the final index, indicators to determine the specific basis is: (1) the index of the coefficient of variation is less than 0.25 ; (2) expert evaluation results through consistency test, $\mathrm{P}<0.01$ and 0.05 ; (3) the selected index mean score above 3.5, that is, to achieve a score as $70 \%$. Coefficient of variation is the ratio of the standard deviation and the average value of each index, the smaller the coefficient of variation, the less the degree of dispersion of the evaluation results of the experts. It is generally believed that the coefficient of variation is greater than or equal to 0.25 , the index of the degree of coordination of the experts is not enough. Coordination coefficient reflects the between group of experts in the expert for the coordinated degree of each index gives advice, with W said the Kendall coefficient of concordance, the significance test of the coordination degree of the hierarchy consistency test. First, the expert questionnaire fill a statistical information, and through the information data entry, in SPSS13. 0 software respectively to set up the database, by SPSS software to describe the data statistics, calculate the indicators at all levels of the mean, standard deviation, through variation coefficient formula that the coefficient of variation. 
Table 3. Primary Indicators Statistical Analysis of Parameters

\begin{tabular}{|c|c|c|c|}
\hline Primary indicators & Mean & $\begin{array}{c}\text { Standard } \\
\text { Deviation }\end{array}$ & $\begin{array}{c}\text { Coefficient } \\
\text { variation }\end{array}$ \\
\hline School sports resources & 5 & 0.000 & 0 \\
\hline $\begin{array}{c}\text { School physical education } \\
\text { process }\end{array}$ & 5 & 0.000 & 0 \\
\hline $\begin{array}{c}\text { School physical education } \\
\text { results }\end{array}$ & 4.6 & 0.507 & 0.1102 \\
\hline $\begin{array}{c}\text { School sports social } \\
\text { environment }\end{array}$ & 3.8 & 0.077 & 0.2039 \\
\hline
\end{tabular}

As shown in Table 3, in the second round of expert investigation, four level index and coefficient of variation were less than 0.25 . The average score is greater than 3.5 , the resources of the school sports and school physical education of the standard deviation and the coefficient of variation was 0 , indicating that experts for the two indicators see exactly the same; after calculating the Kendall coefficient of concordance, can calculate the $p$ value $<0.01$, so a level indicator can determine.

Table 4. Secondary Indicators Statistical Analysis of Parameters

\begin{tabular}{|c|c|c|c|}
\hline Two level index & Mean & $\begin{array}{c}\text { Standard } \\
\text { Deviation }\end{array}$ & $\begin{array}{c}\text { Coefficient } \\
\text { variation }\end{array}$ \\
\hline Sports funds & 4.8 & 0.507 & 0.1056 \\
\hline Field and equipment & 4.86 & 0.403 & 0.828 \\
\hline Physical education teachers & 4.6 & 0.110 & 0.1102 \\
\hline sports management & 4.8 & 0.008 & 0.0086 \\
\hline physical education & 5 & 0 & 0 \\
\hline Spare time sports & 5 & 0 & 0 \\
\hline PE Learning & 5 & 0 & 0 \\
\hline sports culture & 4.2 & 0.414 & 0.0982 \\
\hline Family influence & 3.6 & 0.828 & 0.23 \\
\hline Surrounding environment & 3.5 & 0.516 & 0.1462 \\
\hline
\end{tabular}

In the second round of expert investigation, the results show that the coefficient of variation of 10 second grade indexes were less than 0.25 , and the average number of indicators are more than 3.5. The coefficient of variation of the three indicators of physical education, extracurricular sports and students' physical learning effect, shows that the expert consensus; after calculating the Kendall coefficient of concordance, showed that the $\mathrm{p}$ value $<0.01$; therefore secondary indexes can be determined.

\subsection{Sports Balanced Development Evaluation}

In school physical education balanced development evaluation index system to determine the link in, this study has through Delphi method learned the opinions of the experts, in the determination of the study on the index system of the weighting process, come all the way to combine expert judgment to effectively determine the sequence of importance of every index, to ensure that it does not appear index weights and practical importance of photograph grieve, this evaluation system index weight determination to continue through Delphi for. In the second round of expert questionnaire, ask an expert to each index important degree of scoring, and calculate the average score of each index, and feedback to the experts for the third round of surveys, ask an expert to the second round of the survey index score mean reference no sort of the same grade of each index 
according to the important degree, experts in the anonymous record sorting results and compute the weight of every index.

Table 5. Expert Survey Data of Primary Indicators

\begin{tabular}{|c|c|c|c|c|c|}
\hline Serial & Score & $\begin{array}{c}\text { sum of } \\
\text { square }\end{array}$ & weight & S & \multirow{2}{*}{ W } \\
\hline A1 & 57 & 3249 & 0.377 & & \multirow{2}{*}{0.806} \\
\cline { 1 - 4 } A2 & 42 & 1734 & 0.278 & \multirow{2}{*}{906.75} & \\
\hline A3 & 37 & 1369 & 0.245 & & \\
\hline A4 & 15 & 225 & 0.100 & & \\
\hline
\end{tabular}

Then first by using Excel software to calculate the numerical and the deviation square and $\mathrm{S}=906$. 75 , Kendall coefficient of concordance $\mathrm{W}=0.806 \mathrm{~W}$ value greater than 0.8 , illustrate the consistency of the evaluation results of the experts was higher; square value $\mathrm{X} 2=36.7$, by look-up table shows significant at 0.01 level of chi square value was 11.3 ; finally, because the data generated by the chi square value is 36.7 , so the $\mathrm{W}$ value has reached a significant level, indicating that the experts for the consistency of a grade index evaluation is very strong.

The goal of school physical education process is one of the main objectives of the school physical education, so it is an important part of the evaluation of the balanced development of school sports. School physical education includes three sub targets: first of all, the management of school physical education is a fundamental part of the school physical education, scientific and effective management is a necessary prerequisite for normal school sports work; secondly, curriculum and teaching are two main parts in Chinese sports schools. The only differences between the two are associated, for physical education curriculum contents need through teaching activities to be implemented, so the sports teaching is another key factor in evaluation of school physical education process of; finally, the extracurricular sports is part of the school extracurricular education, school sports are also a component. Extracurricular physical education is an important way to realize the goal of school physical education. It mainly includes the form and content of class exercises, class sports activities, after-school sports training and competition. The school sports and school physical education process should be relative, both are part of the total goal of School Physical Education: the main goal of physical education activities is to enhance the physical fitness of students, to cultivate students' interest in sports, skills, and promote the all-round development of the students, so the school sports results of the first to be reflected from the students, the students interest in sports performance and the degree of love, students are the important content of students' learning results on the sports knowledge and skills in sports classroom learning and mastering; individual learning results are microscopic evaluation, if from the view of whole school evaluation perspective, development, create a school campus sports atmosphere sports atmosphere and school-based physical education curriculum, are also through sports days and months multiplying can be gradually formed, the condensation of the physical education teachers And students' sweat and labor, these are the components of the campus sports culture, so campus sports culture is also one of the components of the results of school sports.

Table 6. Evaluation Index of the Sports Balanced Development

\begin{tabular}{|c|c|}
\hline primary indicators & secondary indicators \\
\hline \multirow{3}{*}{ Resource allocation A1 } & Sports funds B1 \\
$(0.3774)$ & $(0.500)$ \\
\cline { 2 - 2 } & Site and equipment B2 \\
& $(0.3214)$ \\
\cline { 2 - 2 } & Physical education teacher B3 \\
\hline
\end{tabular}




\begin{tabular}{|c|c|}
\hline & $(0.1786)$ \\
\hline \multirow{3}{*}{$\begin{array}{c}\text { School physical education } \\
\text { process A2 }\end{array}$} & $\begin{array}{c}\text { School sports management B4 } \\
(0.4667)\end{array}$ \\
\cline { 2 - 2 }$(0.2781)$ & $\begin{array}{c}\text { Physical education B5 } \\
(0.3667)\end{array}$ \\
\cline { 2 - 2 } & $\begin{array}{c}\text { After-school sports B6 } \\
(0.1667\end{array}$ \\
\hline \multirow{2}{*}{$\begin{array}{c}\text { School physical education } \\
\text { results A3 } \\
(0.2450)\end{array}$} & $\begin{array}{c}\text { Student sports effect B7 } \\
(0.6667)\end{array}$ \\
\cline { 2 - 2 } School sports social environment & Campus sports culture B8 \\
A4 & $(0.3333)$ \\
\cline { 2 - 2 }$(0.1000)$ & $\begin{array}{c}\text { Family influenceB9 } \\
(0.6667)\end{array}$ \\
\cline { 2 - 2 } & $\begin{array}{c}\text { External environmentB10 } \\
(0.3333)\end{array}$ \\
\hline
\end{tabular}

\section{Conclusions}

This paper consists of the balanced development of school physical education theory construction of evaluation index system of the program began, further introduces the general principle and method of constructing principles, the evaluation index system of the balanced development of school physical education, and on the basis of the balanced development of school physical education evaluation index system in the construction process of the index system, the main use of Delphi and method statistics, first through Delphi method through expert questionnaire to collect the opinions of the experts, and then uses the survey data of 3 round expert SPSS statistical software was analyzed and calculated the corresponding results, according to the expert survey index of indicator system was evaluated and screened weight coefficient. The evaluation index system of the balanced development of school physical education should follow the principles of scientific principles, the principle of comprehensiveness, the principle of sensitivity, operability and dynamic.

Anyway, balanced development of school physical education is a long-term, gradual process, so the evaluation content of balanced development of school physical education will change constantly with the development of the progress of the society and school sports, school sports evaluation index system also needs to continue to be updated and revised, hope to have more and more scholars on the balanced development of school physical education, from different levels and angles of theory and practice more in-depth exploration, which is under the realistic background of the balanced development of education, promote the balanced development of school physical education in our country to contribute.

\section{References}

[1] W. Dai and L. Fan, "Discussion about the Pros and Cons and Recommendations for Multimedia Teaching in Local Vocational Schools", Physics Procedia, vol. 33, (2012), pp. 1144-1148.

[2] R. Khansa, "Teachers' Perceptions toward School Counselors in Selected Private Schools in Lebanon", Procedia - Social and Behavioral Sciences, vol. 185, (2015), pp. 381-387.

[3] C. Krstev, and A. Trtovac, "Teaching Multimedia Documents to LIS Students", The Journal of Academic Librarianship, vol. 40, no. 2, (2014), pp. 152-162.

[4] S. J. Hua and L. Hong, "Explore the Effective Use of Multimedia Technology in College Physics Teaching", Energy Procedia, vol. 17, (2012), pp. 1897-1900.

[5] R. A. Sabella, "School counselors perceived importance of counseling technology competencies", Computers in Human Behavior, vol. 26, (2010), pp. 609-617.

[6] N. R. Mastroleo and R. Turrisi, "Examination of posttraining supervision of peer counselors in a motivational enhancement intervention to reduce drinking in a sample of heavy-drinking college students", Journal of Substance Abuse Treatment, vol. 39, (2010), pp. 289-297. 
[7] Z. Huang and M. Benyoucef, "From e-commerce to social commerce: A close look at design features", Electronic Commerce Research and Applications, vol. 12, no. 4, (2013), pp. 246-259.

[8] C. Zhang and X. Chen, "Use of Multimedia in Gross Infective Pathogen Experimental Teaching", Procedia Engineering, vol. 37, (2012), pp. 64-67.

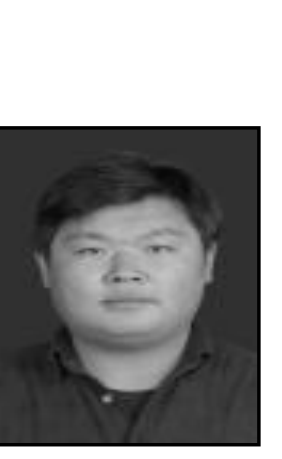

\section{Authors}

Xi Benyu, 1984.10 Xi'an, Shaanxi, P. R. China, Current position, grades: the lecturer of College of educational science, Xi' an Jiaotong University City College, Xi'an, China, Scientific interest: His research interest theory of sports pedagogy and training, Publications: more than 8 papers published, Experience: $\mathrm{He}$ has teaching experience of 5 years, has completed four scientific research projects

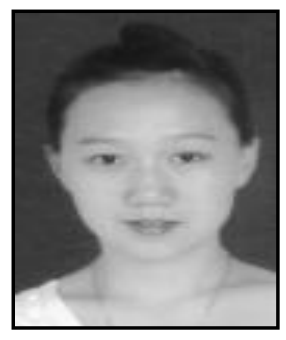

Gong Lina, 1985.5, Xi' an, Shaanxi, P. R. China, Current position, grades: The lecturer of College of educational science, Xi'an Physical Education University Xi'an, China, Scientific interest: Her research interest theory of sports pedagogy and training, Publications: more than 5 papers published, Experience: Her has teaching experience of 10 years, has completed five scientific research projects. 
International Journal of Database Theory and Application Vol.9, No.9 (2016) 